\title{
Killip classification and baseline heart rate can be used to predict streptokinase-induced hypotension in acute myocardial infarction
}

\author{
KARNIZA KHALID ${ }^{1,2}$, RAJA ELINA AHMAD ${ }^{1}$, SZE YEE LUI ${ }^{3}$ and IDA ZALIZA ZAINOL ABIDIN ${ }^{3}$ \\ ${ }^{1}$ Department of Physiology, Faculty of Medicine, University of Malaya, 50603 Kuala Lumpur; ${ }^{2}$ Clinical Research Centre, \\ and ${ }^{3}$ Department of Emergency and Trauma, Hospital Tuanku Fauziah, Kangar, 01000 Perlis, Malaysia
}

Received April 8, 2021; Accepted May 20, 2021

DOI: $10.3892 /$ wasj.2021.110

\begin{abstract}
Streptokinase is the thrombolytic therapy of choice for acute myocardial infarction in hospitals without cardiac facilities. Streptokinase-induced hypotension is one of the common adverse drug reactions and is usually observed within $30 \mathrm{~min}$ of an intravenous streptokinase infusion. The present study aimed to identify predictive clinical parameters for the development of hypotension in patients with acute myocardial infarction. The present retrospective study involved data transcription from National Indicator Approach (NIA) records of acute myocardial infarction recorded between 2015 to 2018. Multivariate analysis was performed to evaluate potential predictors of streptokinase-induced hypotension and to determine the association between selected clinical variables in patients with streptokinase-induced hypotension. The present study included a total of 412 patients with acute myocardial infarction administered streptokinase. The majority $(n=258,62.6 \%)$ did not develop any complication from the therapy, whereas 109 (26.5\%) developed hypotension at 18.5 (interquartile range, 10.00) min from the initiation of therapy. Multiple logistic regression analysis revealed that with every one-unit increment in the baseline heart rate, the risk of hypotension was reduced by $2.6 \%$. Additionally, patients classified as Killip class III and IV were at an increased risk (5-fold) of developing hypotension as compared with those classified as Killip I. Therefore, as demonstrated herein, these predictive factors may assist clinicians in identifying susceptible individuals and may encourage vigilance when delivering streptokinase therapy.
\end{abstract}

\section{Introduction}

Streptokinase is a protein harvested from $\beta$-hemolytic Streptococci (1). It has been established that streptokinase

Correspondence to: Dr Karniza Khalid, Clinical Research Centre, Hospital Tuanku Fauziah, Jalan Tun Abdul Razak, Kangar, 01000 Perlis, Malaysia

E-mail: karniza.khalid@moh.gov.my

Key words: streptokinase, hypotension, blood pressure determination, blood viscosity, logistic models possesses fibrinolytic properties through the activation of plasma plasminogen via fibrin-dependent and fibrin-independent pathways (2). Therefore, streptokinase has been used as one of the therapeutic modalities in the treatment of thrombo-embolic diseases, such as pulmonary embolism and acute myocardial infarction. Despite its proven efficacy, hypotension is a common side-effect observed with intravenous streptokinase therapy in patients with acute myocardial infarction.

Hypotension with streptokinase therapy is commonly attributed to the non-specific effect of streptokinase, as it not only activates the clot-derived plasminogen, but also the circulating plasma plasminogen, resulting in systemic fibrinolysis and fibrinogenolysis (3); thus, its uses is associated with the occurrence of hypotension and bleeding tendencies (4-6). Streptokinase-induced hypotension has been the subject of interest since the 1970s. To date, the exact mechanisms responsible for its development remain obscure (7).

Therefore, the present study aimed to predict the occurrence of streptokinase-induced hypotension from selected baseline clinical parameters in order to aid the identification of patients with acute myocardial infarction who are at risk of developing hypotension during streptokinase therapy.

\section{Patients and methods}

Patients. This cross-sectional study involved data transcription from hospital National Indicator Approach (NIA) records for acute myocardial infarction (AMI) cases recorded at the Emergency and Trauma Department of Hospital Tuanku Fauziah (HTF), Perlis, Malaysia from 2015 to 2018. Acute myocardial infarction was diagnosed in the presence of typical signs and symptoms, the presence of suggestive electrocardiographic changes, and positive cardiac markers (including, but not limited to troponin test).

ST-elevation acute myocardial infarction cases receiving intravenous streptokinase were included in the present study. On the other hand, patients requiring prior hemodynamic stabilisation prior to streptokinase administration (i.e., requiring inotropic support or glyceryl trinitrate infusion prior to streptokinase), or prior cardiopulmonary resuscitation were excluded from the study. Information on demographic details (personal identifier, age, sex, race) and acute management details (time of streptokinase delivery and completion, 
side-effects observed during streptokinase and management, and vital signs (serial blood pressure and heart rate recording during streptokinase) were transcribed into a separate data sheet. The Killip classification quantifies the severity of heart failure following acute myocardial infarction as follows: Killip class I refers to no evidence of heart failure; class II refers to signs of suggestive of mild-to-moderate heart failure, such as the presence of S3 gallop and distended jugular veins; class III refers to overt pulmonary edema, and class IV refers to cardiogenic shock (8).

Laboratory results obtained at pre- and immediately post-streptokinase delivery were traced from the digital records of the hospital database. Laboratory data included data for baseline cardiac enzymes, such as creatine kinase (CK)-MB (CKMB), full blood count and renal profile.

Ethical clearance. The present study was registered with the National Medical Research Registry of Ministry of Health, Malaysia NMRR-19-2871-50953 and received ethical clearance from the Medical Research and Ethics Committee (MREC), Ministry of Health Malaysia [KKM/NIHSEC/P19-2421(5)]. Informed consent related to the present study was waived by the Medical Research and Ethics Committee of the Ministry of Health Malaysia. The study only utilized secondary data source and did not involve any form of physical interaction with the patients.

Statistical analyses. Statistical analyses were conducted using IBM SPSS software version 23.0 (IBM Corp.). Descriptive data, such as sex and race were described using percentages and distribution. Numerical data (such as age and baseline hemoglobin) were described as the mean \pm standard deviation; however, skewed data, such as the Troponin-I level, baseline total white blood cell count, baseline platelet count, baseline neutrophil count, baseline urea and baseline creatinine level were described as the median and interquartile range (IQR).

Continuous variables between two groups (hypotensive vs. normotensive) were compared using an independent t-test in data with normal distribution (i.e., age), and with the Mann-Whitney $U$ test in data with skewed distribution (i.e., Troponin-I level, baseline total white blood cell count, baseline platelet count, baseline neutrophil count, baseline urea and baseline creatinine levels).

Pearson's Chi-squared test of independence was performed to determine the association between categorical variables (sex, location myocardial infarction, baseline creatine kinase levels), between patients with hypotension vs. those with no hypotension. For categorical associations not eligible for Pearson's Chi-squared test (by having $>20 \%$ of the expected counts of $<5)$, a corresponding Fisher's exact test was performed (i.e., baseline urea/creatinine ratio).

Repeated measures analysis of variance (ANOVA) with the Greenhouse-Geisser correction was performed to examine the effect of time on systolic and diastolic blood pressure. The Greenhouse-Geisser correction was applied to adjust for the lack of sphericity in repeated measures ANOVA. Multivariate analysis was performed to identify predictive factors contributing to systemic hypotension through simple logistic regression (SLR) and multiple logistic regression (MLR) analyses. MLR was performed using the ENTER method whereby all the variables were included in the modelling regardless of their independent statistical significance. The ENTER method was selected in view of its default option for regression analysis in the majority of statistical software (9). Nagelkerke R squares provides the indication of the amount of variation present in the dependent variable able to be explained by the model. The value ranges from zero (0) to one (1), and commonly described in percentage $(10)$. A probability value (P-value) $<0.05$ was deemed as statistically significant.

\section{Results}

Patients with treatment-related complications. There were 412 patients enrolled in the present study based on the pre-defined criteria. The majority of the patients $(n=258,62.6 \%)$ did not develop any complications from the therapy, whereas the most common adverse drug reactions observed among patients who were treated was hypotension $(n=109,26.5 \%)$, hypertension $(n=16,14.7 \%)$, allergic reaction $(n=11,2.7 \%)$ and bleeding tendencies $(\mathrm{n}=11,2.7 \%)$. The less common complications included bradycardia $(n=5,1.2 \%)$ and arrhythmia $(n=2,0.4 \%)$.

The mean age of the study participants was $59.3 \pm 12.61$ years. The majority were male $(n=303,73.5 \%)$ and of Malay descent $(n=328,79.6 \%)$. Anterior myocardial infarction was more common vs. inferior myocardial infarction (50.1 vs. 41.7\%).

Baseline study parameters. The demographic characteristics and comparison of baseline parameters between patients with streptokinase-induced hypotension and patients with no hypotension from streptokinase therapy are presented in Table I. Only complete datasets with regards to individual variable were included in this descriptive analysis; hence, the cumulative numbers may not add up to the total of 412 patients.

Systolic and diastolic blood pressure. The mean systolic blood pressure recorded at baseline was $138.1 \pm 21.09 \mathrm{mmHg}$ in patients with no hypotension and $131.6 \pm 22.91 \mathrm{mmHg}$ in patients with streptokinase-induced hypotension, $t(357)=2.56, \mathrm{P}=0.011$.

The mean diastolic blood pressure recorded at baseline was $86.7 \pm 15.33 \mathrm{mmHg}$ in patients with no hypotension and $80.4 \pm 15.16 \mathrm{mmHg}$ in patients with streptokinase-induced hypotension, $t(356)=3.46, \mathrm{P}=0.001$.

Fig. 1 presents a graphical comparison of the systolic and diastolic blood pressure variations with time between patients with streptokinase-induced hypotension and no hypotension.

Sub-analysis in patients with streptokinase-induced hypotension. Sub analyses in patients with streptokinase-induced hypotension were performed on 109 subjects. The median duration for patients to develop streptokinase-induced hypotension was at $18.5 \mathrm{~min}$ (IQR, $10.00 \mathrm{~min}$ ) from the initiation of intravenous streptokinase infusion therapy (range, 2.0-60.0 min).

The lowest mean arterial pressure recorded among patients with streptokinase-induced hypotension was $54.6 \pm 9.72 \mathrm{mmHg}$. The lowest recorded mean systolic blood pressure among patients with streptokinase-induced hypotension was $75.8 \pm 10.81 \mathrm{mmHg}$, whereas the lowest recorded mean diastolic blood pressure was $42.9 \pm 10.37 \mathrm{mmHg}$, and the mean heart rate recorded at the lowest blood pressure drop among this group of patients was $76.5 \pm 20.74$ beats per minute. 
Table I. Baseline characteristics and parameters of study population.

\begin{tabular}{|c|c|c|c|c|c|}
\hline \multirow[b]{2}{*}{ Variable(s) } & \multicolumn{2}{|c|}{ Hypotension } & \multicolumn{2}{|c|}{ No hypotension } & \multirow[b]{2}{*}{ P-value } \\
\hline & Mean (SD) & $\mathrm{n}(\%)$ & Mean (SD) & $\mathrm{n}(\%)$ & \\
\hline $\mathrm{Age}^{\mathrm{a}}$ & $60.6(10.79)$ & $106(29.2)$ & $58.7(13.27)$ & $257(70.8)$ & 0.158 \\
\hline $\operatorname{Sex}^{b}$ & & & & & 0.100 \\
\hline Male & & $84(27.7)$ & & $219(72.3)$ & \\
\hline Female & & $24(38.1)$ & & $39(61.9)$ & \\
\hline Race $^{c}$ & & & & & 0.103 \\
\hline Malay & & $99(30.2)$ & & $229(69.8)$ & \\
\hline Chinese & & $3(12.5)$ & & $21(87.5)$ & \\
\hline Indian & & $3(33.3)$ & & $6(66.7)$ & \\
\hline Others & & $3(60.0)$ & & $2(40.0)$ & \\
\hline Location $\mathrm{MI}^{\mathrm{b}}$ & & & & & 0.090 \\
\hline Anterior & & $47(25.5)$ & & $137(74.5)$ & \\
\hline Inferior & & $52(34.0)$ & & $101(66.0)$ & \\
\hline Baseline $\mathrm{CK}^{\mathrm{b}}(\mathrm{U} / \mathrm{l})$ & & & & & 0.970 \\
\hline Normal $(<171)$ & & $52(31.1)$ & & 115 (68.9) & \\
\hline Raised ( $\geq 171)$ & & $43(30.9)$ & & $96(69.1)$ & \\
\hline Baseline SBP & $131.6(22.91)$ & & $138.1(21.09)$ & & $0.011^{\mathrm{f}}$ \\
\hline Baseline DBP & $80.4(15.16)$ & & $86.7(15.33)$ & & $0.001^{\mathrm{f}}$ \\
\hline Baseline Trop-I ${ }^{\mathrm{d}}(\mathrm{ng} / \mathrm{ml})$ & $2.0(0.96)$ & $18(32.1)$ & $2.3(1.10)$ & $38(67.9)$ & 0.329 \\
\hline Baseline $\mathrm{TWBC}^{\mathrm{d}}\left(\mathrm{x} 10^{3} / \mu \mathrm{l}\right)$ & $12.1(4.20)$ & $95(28.5)$ & $11.5(5.51)$ & $238(71.5)$ & 0.465 \\
\hline Baseline $\mathrm{Hb}^{\mathrm{a}}(\mathrm{g} / \mathrm{dl})$ & $13.8(1.86)$ & $95(28.5)$ & $14.2(2.02)$ & $238(71.5)$ & 0.069 \\
\hline Baseline Plt count $^{\mathrm{d}}\left(\mathrm{x} 10^{3} / \mu \mathrm{l}\right)$ & $277.0(93.00)$ & $95(28.7)$ & $258.5(95.0)$ & $236(71.3)$ & 0.240 \\
\hline Baseline neutrophil count ${ }^{\mathrm{d}}\left(\mathrm{x} 10^{3} / \mu \mathrm{l}\right)$ & $7.4(5.19)$ & $95(28.5)$ & $6.9(4.69)$ & $238(71.5)$ & 0.601 \\
\hline Baseline urea $^{\mathrm{d}}(\mathrm{mmol} / \mathrm{l})$ & $5.2(3.20)$ & $94(30.7)$ & $4.6(2.40)$ & $212(69.3)$ & 0.107 \\
\hline Baseline creatinine $^{\mathrm{d}}(\mu \mathrm{mol} / \mathrm{l})$ & $107.0(40.0)$ & $94(30.7)$ & $96.0(32.00)$ & $212(69.3)$ & 0.142 \\
\hline Baseline Ur/Creat ratio ${ }^{\mathrm{c}, \mathrm{e}}$ & & & & & 0.793 \\
\hline Pre-renal & & $4(40.0)$ & & $6(60.0)$ & \\
\hline Renal & & $11(30.6)$ & & $25(69.4)$ & \\
\hline Post-renal & & $5(38.5)$ & & $8(61.5)$ & \\
\hline
\end{tabular}

Only complete datasets with regards to individual variable and grouping were included in this descriptive analysis, hence the cumulative frequency may not add up to the total of 412 patients (as observed with age, sex, race, location MI, baseline CK, baseline SBP and DBP, baseline troponin-I, baseline TWBC, baseline Plt, baseline neutrophil count, baseline urea, baseline creatinine, and baseline urea/creatinine ratio). The percentage calculated was also based on the total number of complete dataset, hence missing data were not counted for. ${ }^{2} D a t a$ were analyzed using an independent t-test; 'bata were analyzed using Pearson's Chi-squared test of independence; 'data were analyzed using Fisher's exact test; ${ }^{\mathrm{d}}$ data were analyzed using the Mann-Whitney U test (presented as the median and interquartile range). ${ }^{\mathrm{e}} \mathrm{Hydration}$ status was assessed quantitatively through the increased urea/creatinine ratio with a urea level $>10 \mathrm{mmol} / \mathrm{l}$. Pre-renal features may suggest dehydration. ${ }^{\mathrm{f}}<0.05$, indicates statistical significance. CK, creatine kinase; Trop-I, Troponin-I; SBP, systolic blood pressure; DBP, diastolic blood pressure.

Similarly, both groups of patients (with and without hypotension) received the median cumulative dosage of $33.9 \mathrm{ml}$ (IQR, $18.30 \mathrm{ml}$; range, 3.7-109.8 ml) of intravenous streptokinase, which equates to $508,500 \mathrm{IU}$ of streptokinase.

The results of repeated measures ANOVA between the systolic blood pressure, diastolic blood pressure and heart rate at baseline, and at 10,20 and 30 min during streptokinase therapy in patients with streptokinase-induced hypotension are presented in Table II.

Predictors of streptokinase-induced hypotension. SLR was conducted to determine the odds of each selected variable in predicting streptokinase-induced hypotension independently, whereas MLR was performed done to determine the optimal predictive model with a combination of variables that could positively predict the occurrence of streptokinase-induced hypotension (Table III). Only complete datasets with regards to individual variable were included in this descriptive analysis, hence the cumulative numbers may not add up to the total of 412 patients.

MLR using the ENTER method revealed that Killip categories and baseline heart rate were predictive to the development of hypotension after adjusting for age, sex, baseline CK and baseline systolic and diastolic blood pressures. The Nagelkerke R-squared measures determined that $12.9 \%$ of the variation in the dependent variable can be explained by the model. 
Table II. Changes in blood pressure in patients with hypotension receiving streptokinase.

\begin{tabular}{lcccccr}
\hline & \multicolumn{2}{c}{ Pre-STK } & & \multicolumn{3}{c}{ During STK therapy } \\
\cline { 2 - 3 } Variable(s) & Baseline mean (SEM) & & 10-min mean (SEM) & 20-min mean (SEM) & 30-min mean (SEM) & P-value \\
\hline $\mathrm{SBP}^{\mathrm{a}}(\mathrm{mmHg})$ & $130.7(2.33)$ & & $108.5(3.05)$ & $100.0(2.75)$ & $114.5(2.24)$ \\
$\mathrm{DBP}^{\mathrm{a}}(\mathrm{mmHg})$ & $80.0(1.58)$ & & $66.7(2.24)$ & $59.9(1.97)$ & $60.001^{\mathrm{b}}$ \\
$\mathrm{HR}^{\mathrm{a}}(\mathrm{bpm})$ & $78.4(1.96)$ & & $77.6(1.91)$ & $77.8(2.08)$ & $(1.41)$ & $<0.001^{\mathrm{b}}$ \\
\hline
\end{tabular}

${ }^{a}$ Data were analyzed using a repeated measures ANOVA and the Greenhouse-Geisser correction. HR, heart rate; DBP, diastolic blood pressure;



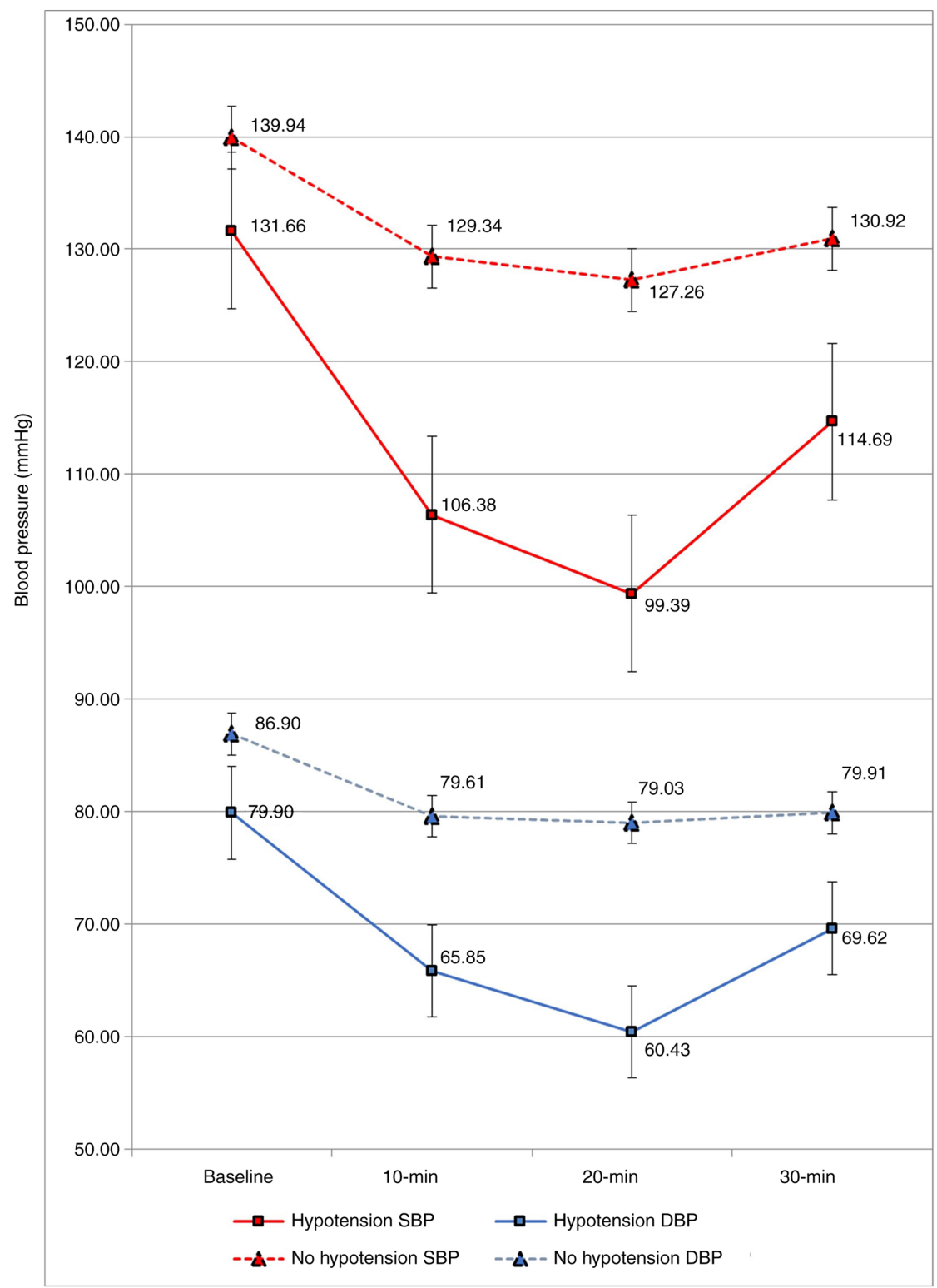

Figure 1. Systolic and diastolic blood pressure variations with time in patients with hypotension vs. those with no hypotension. SBP, systolic blood pressure; DBP, diastolic blood pressure.

It was found that with every one-unit increment in the heart rate, the risk of hypotension was reduced by $2.6 \%$. Additionally, patients classified as Killip III and IV had increased odds for the development of hypotension by $\sim 5$-fold as compared to those classified as Killip I.

\section{Discussion}

Fibrinolytic therapy is secondary to the gold standard percutaneous coronary intervention (PCI) for the management of patients with acute myocardial infarction (11). Despite being 


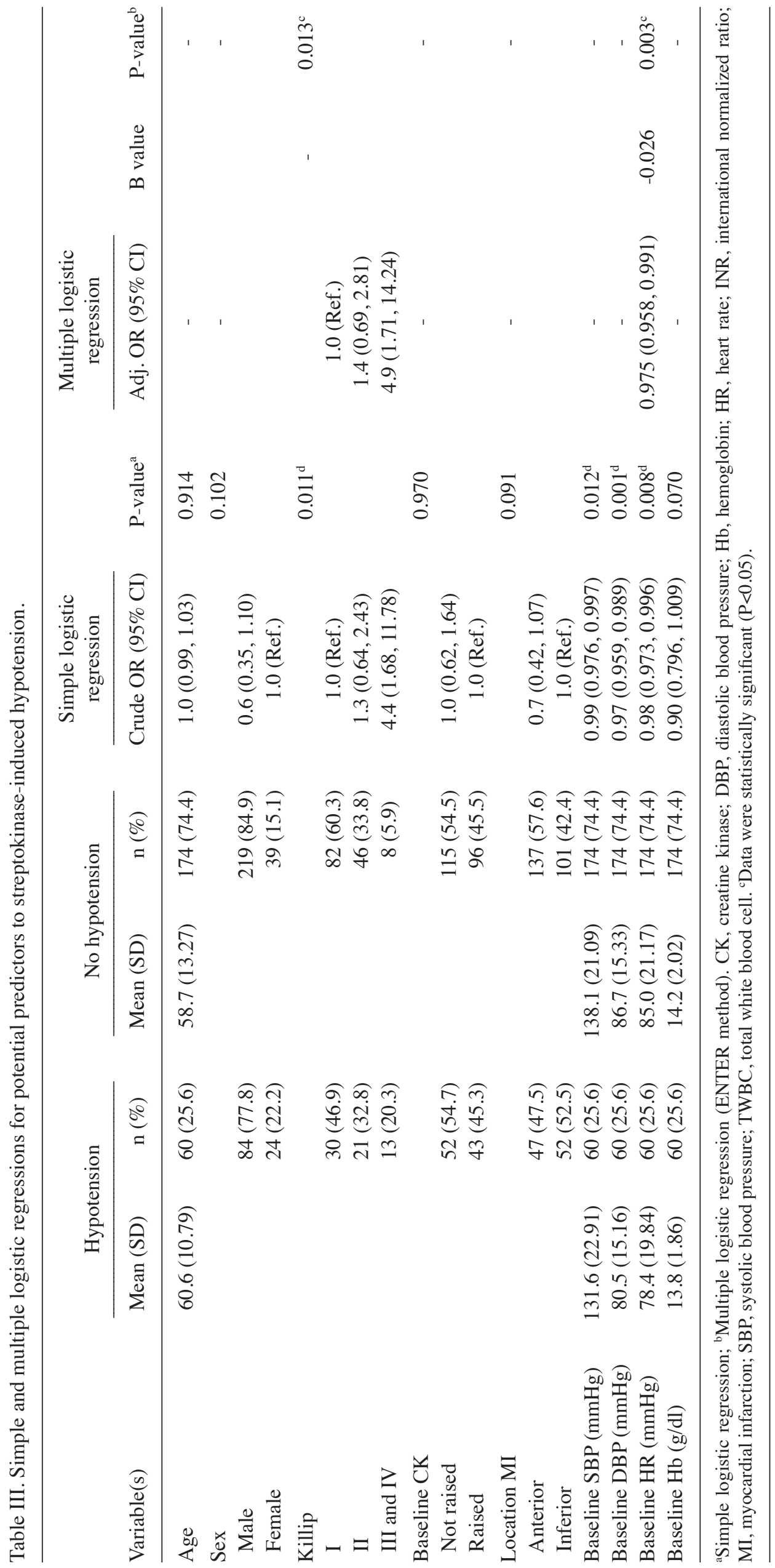


the gold standard treatment approach for myocardial infarction, the availability of PCI service is limited to cardiac referral centers, particularly in developing countries. The majority of public healthcare institutes without cardiac facilities still utilize first generation thrombolytics, such as streptokinase, as the primary mode of management in patients with acute myocardial infarction.

The present study determined that the hypotension observed during the intravenous infusion of streptokinase was a specific side-effect of the therapy in view of the comparable baseline demographic data and pertinent cardiac biomarkers, such as CKMB and Troponin-I, between patients with hypotension and without hypotension.

It was found that the onset of streptokinase-induced hypotension at occurred $18.5 \mathrm{~min}$ (IQR, $10.00 \mathrm{~min}$ ) from the initiation of intravenous streptokinase therapy, synonymous to other studies that have reported a mean of 15-30 min (4,12-14).

Previous studies have also suggested that streptokinaseinduced hypotension is a dose-related phenomenon (15-17); however, this hypothesis could not be supported, as the present study found no association between streptokinase cumulative dosages with the lowest documented mean arterial pressure. This finding was also shared by other previous studies that have suggested that an accelerated streptokinase delivery $>20-30 \mathrm{~min}$, instead of the conventional 60-min infusion is equally safe, apart from offering higher coronary reperfusion rates (17-19).

As regards the predictive factors for streptokinase-induced hypotension, the present study found that there were no significant differences in the baseline cardiac-specific biomarkers, such as the CKMB and Troponin-I levels between patients with hypotension and patients with no hypotension. Hence, it could not be proved that the occurrence of hypotension during streptokinase therapy is related to the extent of myocardial injury, as similarly observed by in the study by Pachaï et al (20). Other baseline clinical components that were considered as potential confounding factors to the development of hypotension during intravenous streptokinase therapy include a poor hydration status (15) and inferior infarcts (21). In the present study, the state of hydration was determined from the disproportionate increase in the urea to creatinine ratio, which was insignificant between the two groups in the study population. Additionally, neither anterior nor inferior infarcts, predicted the occurrence of hypotension during streptokinase therapy in the present study; a similar observation was reported in the study by Chau and Choi (21).

The present study did not use other underlying comorbidities in patients, such as diabetes or hypertension as factors in the predictive model, as the present study focused on the immediate side-effects of streptokinase therapy during the acute onset of myocardial necrosis. The presence and level of control of these underlying medical problems were indeed significant risk factors for the development of acute myocardial infarction; however, they have not been proven to independently influence the development of hypotension during streptokinase therapy. However, the present study examined all the measurable determinants of blood pressure, including factors that affect cardiac output and systemic vascular resistance. This included the measurements of heart rate, calculated whole blood viscosity and the assessment of hydration.

To the best of our knowledge, no previous study to date has investigated the potential baseline clinical parameters for the prediction of the development of streptokinase-induced hypotension. The present study revealed two baseline parameters that significantly predicted the occurrence of hypotension in patients with acute myocardial infarction receiving streptokinase therapy, which are the baseline heart rate prior to streptokinase therapy and the Killip classification. The Killip classification was first introduced in 1967 (22) as a tool in clinical practice to assess the severity of heart failure following myocardial infarction. The four stages of Killip classification ranges from no clinical signs of heart failure to overt cardiogenic shock. Secondly, the lower baseline heart rate was also determined to be predictive of hypotension during streptokinase therapy. The heart rate is part of the hemodynamic markers and reflects the autonomic function of the heart and residual myocardial reserve following myocardial injury. Therefore, it may also affect blood pressure through the modulation in cardiac output. This finding is in agreement with that of the study by Lew et al (12) who found that patients with a compromised hemodynamic status had a higher risk of developing hypotension during streptokinase therapy.

Therefore, these predictive factors may assist clinicians in identifying susceptible individuals and may encourage vigilance when delivering streptokinase in patients with acute myocardial infarction.

In conclusion, the findings of the present study underscore the importance of clinical vigilance during streptokinase therapy. The anticipation of hypotension in susceptible individuals prompts caution during clinical management to prevent further hypoperfusion in the acutely ischemic myocardium. A hypotensive episode during acute myocardial infarction could further extend the rate of myocardial necrosis and affect the non-ischemic myocardium as well, particularly if the areas are supplied by collaterals of stenotic coronary arteries.

\section{Acknowledgements}

The authors would like to thank the Director General of Health Malaysia for his permission to publish this article.

\section{Funding}

No funding was received.

\section{Availability of data and materials}

The datasets used and/or analyzed during the current study are available from the corresponding author on reasonable request.

\section{Authors' contributions}

The study was conceptualized by KK, REA, SYL and IZZA. KK and SYL performed the data collection and statistical analysis, prepared the tables and drafted the manuscript. REA, SYL and IZZA commented on the manuscript and tables. KK and SYL confirm the authenticity of all the raw data. All authors have read and approved the final manuscript. 


\section{Ethics approval and consent to participate}

The present study was registered with the National Medical Research Registry of Ministry of Health, Malaysia (NMRR-19-2871-50953 and received ethical clearance from the Medical Research and Ethics Committee (MREC), Ministry of Health Malaysia [KKM/NIHSEC/P19-2421(5)]. Informed consent related to the present study was waived by the Medical Research and Ethics Committee of the Ministry of Health Malaysia. The study only utilized secondary data source and did not involve any form of physical interaction with the patients.

\section{Patient consent for publication}

Not applicable.

\section{Competing interests}

The authors declare there that they have no competing interests.

\section{References}

1. Adivitiya and Khasa YP: The evolution of recombinant thrombolytics: Current status and future directions. Bioengineered 8 331-358, 2017.

2. Banerjee A, Chisti Y and Banerjee UC: Streptokinase-a clinically useful thrombolytic agent. Biotechnol Adv 22: 287-307, 2004.

3. Grierson DS: Clinical pharmacology of streptokinase. In: Acute Coronary Care. Springer, New York, NY, pp67-74, 1985.

4. Gemmill JD, Hogg KJ, Douglas JT, Dunn FG, Lowe GD, Rae AP and Hillis WS: The incidence and mechanism of hypotension following thrombolytic therapy for acute myocardial infarction with streptokinase-containing agents-lack of relationship to pretreatment streptokinase resistance. Eur Heart J 14: 819-825, 1993.

5. Kunamneni A, Abdelghani TT and Ellaiah P: Streptokinase-The drug of choice for thrombolytic therapy. J Thromb Thrombolysis 23: 9-23, 2007

6. Aslanabadi N, Safaie N, Talebi F, Dousti S and Entezari-Maleki T: The streptokinase therapy complications and its associated risk factors in patients with acute ST elevation myocardial infarction. Iran J Pharm Res 17 (Suppl 1): S53-S63, 2018.

7. Kumolosasi E, Wei WS and Wee CE: The use of thrombolytic agents in acute myocardial infarction (AMI) patients. Int J Pharm Pharm Sci 5: 63-67, 2013

8. El-Menyar A, Zubaid M, Almahmeed W, Sulaiman K, Alnabti A, Singh R and Al Suwaidi J: Killip classification in patients with acute coronary syndrome: Insight from a multicenter registry. Am J Emerg Med 30: 97-103, 2012.
9. Ranganathan P, Pramesh CS and Aggarwal R: Common pitfalls in statistical analysis: Logistic regression. Perspect Clin Res 8: 148-151, 2017.

10. Nagelkerke NJD: A note on a general definition of the coefficient of determination. Biometrika 78: 691-692, 1991.

11. Nakamura M: Angiography is the gold standard and objective evidence of myocardial ischemia is mandatory if lesion severity is questionable. -Indication of PCI for angiographically significant coronary artery stenosis without objective evidence of myocardial ischemia (Pro)-. Circ J 75: 204-210, 217, 2011.

12. Lew AS, Laramee P, Cercek B, Shah PK and Ganz W: The hypotensive effect of intravenous streptokinase in patients with acute myocardial infarction. Circulation 72: 1321-1326, 1985.

13. Battershill PE, Benfield P and Goa KL: Streptokinase. A review of its pharmacology and therapeutic efficacy in acute myocardial infarction in older patients. Drugs Aging 4: 63-86, 1994.

14. Carnemolla R and Muzykantov VR: Vascular targeting of antithrombotic agents. IUBMB Life 63: 632-639, 2011.

15. Ghaffari S, Kazemi B and Golzari IG: Efficacy of a new accelerated streptokinase regime in acute myocardial infarction: A double blind randomized clinical trial. Cardiovasc Ther 31: 53-59, 2013.

16. Srimahachota S, Sangwatanaroj S, Boonyaratavej S, Suitichaiyakul T and Ngarmukos P: Efficacy of rapid infusion of streptokinase in patients with acute myocardial infarction. J Med Assoc Thai 83: 8-12, 2000.

17. Tatu-Chițoiu G, Dorobanţu M, Teodorescu C, Craiu E, Vintilǎ M, Minescu B, Burghină D, Stamate S, Serban L, Protopopescu T, et al: Accelerated streptokinase in ST-elevation myocardial infarction-a romanian (ASK-ROMANIA) multicenter registry. Int J Cardiol 122: 216-223, 2007.

18. Herlitz J, Hartford M, Aune S and Karlsson T: Occurrence of hypotension during streptokinase infusion in suspected acute myocardial infarction, and its relation to prognosis and metoprolol therapy. Am J Cardiol 71: 1021-1024, 1993.

19. Koren G, Weiss AT, Ben-David Y, Hasin Y, Luria MH and Gotsman MS: Bradycardia and hypotension following reperfusion with streptokinase (Bezold-Jarisch reflex): A sign of coronary thrombolysis and myocardial salvage. Am Heart J 112: 468-471, 1986

20. Pachaï A, Erlendsson AK and Brandslund I: Streptokinase, complement activation and hypotension. APMIS 105: 650-654, 1997.

21. Chau HW and Choi KK: Efficacy and safety of tenectaplase versus streptokinase in treating st-elevation myocardial infarction patients in Hong Kong: A four-year retrospective review in Queen Elizabeth Hospital. Hong Kong J Emerg Med 20: 359-363, 2013.

22. Killip T III and Kimball JT: Treatment of myocardial infarction in a coronary care unit. A two year experience with 250 patients. Am J Cardiol 20: 457-464, 1967. 